\title{
Capsule Commentary on Haas et al., Primary Care Providers' Beliefs and Recommendations and Use of Screening Mammography by their Patients
}

\author{
Daniel Meyer, MD \\ Medical College of Wisconsin, Milwaukee, WI, USA. \\ JGenInternMed32(4):473 \\ DOI: $10.1007 / \mathrm{s} 11606-017-3986-1$ \\ (c) Society of General Internal Medicine 2017
}

B reast cancer screening recommendations from the US Preventive Service Task Force (USPSTF) were updated in 2009. ${ }^{3}$ Reports are conflicting as to whether mammography use has changed in light of those changes. ${ }^{2,4}$ Haas et al. ${ }^{1}$ investigated whether primary care providers' beliefs regarding the effectiveness of screening mammography influenced utilization by their patients.

Haas et al. ${ }^{1}$ stratified patients into age groups that parallel USPSTF guidelines. The most striking association was the increased use of mammograms by patients of providers expressing uncertainty about the utility of mammography in reducing breast cancer mortality. This was found among all age groups, but was only statistically significant among the 49-74-year age group. Additionally, there was discordance between provider belief in mammography effectiveness and patient receipt of mammograms. In women aged $75+$ years, patients of providers who believed mammography to be 'very effective' had an OR of only 0.63 (of receiving a mammogram) compared to providers believing mammography to be 'somewhat effective'. The authors conclude that physician beliefs were largely independent of patient breast cancer screening.

While the study indicates that it has screening utilization data from 2011 and 2014, it states that the majority of patients had 24 months of follow-up. Such a short time frame is inadequate for assessing the evolution of patients' attitudes and beliefs. Sprague et al. ${ }^{2}$ demonstrated a modest decrease in the rates of mammography among women aged 40-49 years from 2009 to 2011 in Vermont (45.3\% to 41.6\%), which

coincides with USPSTF 2009 guideline changes. Another limitation of the study by Haas et al. is that it incorporates patient data only to the level of the Charlson comorbidity score. Providers may describe their beliefs and attitudes regarding average-risk patients, but the study does not confirm whether the studied patient population is average-risk.

In seeking to provide evidence-based care, providers and administrators need to appreciate the complexity of breast cancer screening and the multitude of factors that influence patient behavior ${ }^{5}$. Once motiving factors are better understood, providers will be better able to facilitate behavioral changes aligned with best practices.

Corresponding Author: Daniel Meyer, MD; Medical College of Wisconsin, Milwaukee, WI, USA (e-mail: dmeyer@mcw.edu).

\section{Compliance with Ethical Standards:}

Conflict of Interest: The author has no conflicts of interest with this article.

\section{REFERENCES}

1. Haas et al. Primary Care Providers' Beliefs and Recommendations and Use of Screening Mammography by their Patients. J Gen Intern Med 2017 doi:10.1007/s11606-016-3973-y.

2. Sprague BL, Bolton KC, Mace JL, et al. Registry-based study of trends in breast cancer screening mammography before and after the 2009 U.S. Preventive Services Task Force recommendations. Radiology. 2014;270(2):354-361.

3. Screening for breast cancer: U.S. Preventive Services Task Force recommendation statement. Ann Intern Med. 2009; 151(10):716-726, W-236.

4. Pace LE, He Y, Keating NL. Trends in mammography screening rates after publication of the 2009 US Preventive Services Task Force recommendations. Cancer. 2013;119(14):2518-2523.

5. Zapka JG, Taplin SH, Solberg LI, Manos MM. A framework for improving the quality of cancer care: the case of breast and cervical cancer screening. Cancer Epidemiol Biomarkers Prev. 2003;12(1):4-13. 